\title{
Thermodynamic Assessment of Liquid Fe-Ni-C Alloy Using Modified Quasichemical Model
}

\author{
MIN-KYU PAEK (1) ${ }^{1,5}$ JUNMO JEON, ${ }^{1}$ MANAS PALIWAL, ${ }^{2}$ \\ ARIJIT BISWAS, ${ }^{3}$ DANIEL LINDBERG,${ }^{1}$ and JONG-JIN PAK ${ }^{4}$ \\ 1.-Department of Chemical and Metallurgical Engineering, Aalto \\ University, 02150 Espoo, Finland. 2.-Department of Material Science and Engineering, Indian \\ Institute of Technology Gandhinagar, Gandhinagar, Gujarat 382355, India. 3.-Division of Ferro \\ Alloys and Minerals, Tata Steel, Jamshedpur 831009, India. 4.-Department of Materials Science \\ and Chemical Engineering, Hanyang University, ERICA, Ansan 15588, Korea. \\ 5.-e-mail: min.paek@aalto.fi
}

\begin{abstract}
The liquid solution properties in the $\mathrm{Fe}-\mathrm{Ni}$ and $\mathrm{Fe}-\mathrm{Ni}-\mathrm{C}$ systems have been thermodynamically assessed using the modified quasichemical model in the pair approximation. The asymmetry of the Fe-Ni and Fe-Ni-C alloy melts was verified by measuring the melting points of $\mathrm{Fe}-\mathrm{Ni}$ binary alloys on the Ni-rich side, and the $\mathrm{C}$ solubility limit in $\mathrm{Fe}-\mathrm{Ni}$ melt over the entire concentration range at $1500^{\circ} \mathrm{C}$ and $1600^{\circ} \mathrm{C}$. The solubility minimum of $\mathrm{C}$ and the maximum partial enthalpy of mixing of $\mathrm{C}$ in the ternary Fe-Ni-C system occurred at near the maximum short-range ordering composition of the binary Fe-Ni liquid solution. The extrema of the partial properties of $\mathrm{C}$ in the ternary $\mathrm{Fe}-\mathrm{Ni}$-C system were successfully reproduced with only one constant adjustable ternary parameter.
\end{abstract}

\section{INTRODUCTION}

Recently, high-nickel-containing alloys have received much attention for the production of superalloys and high-entropy alloys because they can enhance the mechanical properties of the alloys, such as high corrosion resistance, high-temperature strength, and low thermal expansion properties at a specific Ni concentration. ${ }^{1,2}$ Even though the Ni concentration has been increasing for such special grades of steels and alloys, the information on the liquid solution properties of Ni-based alloys is still scant. Contrary to the recent experimental studies on the ordered/disorder transition of face centered cubic (FCC) solid solution in a low-temperature range, ${ }^{3,4}$ little has been reported on the melting point of the Ni-rich side in the Fe-Ni system since the $1920 \mathrm{~s} .{ }^{5,6}$ In addition, the control of $\mathrm{C}$ content during the refining and heat treatment of high-Nicontaining alloy production is a big issue because the carbon content significantly influences the strength and ductility in the Ni-based alloys by

(Received August 7, 2020; accepted November 2, 2020; published online November 25, 2020) suppressing the dislocation motion. ${ }^{7,8}$ However, the thermodynamic behavior of $\mathrm{C}$ in the ternary $\mathrm{Fe}-\mathrm{Ni}$ $\mathrm{C}$ liquid solution has not been clearly described at high temperatures.

The demand for high-purity raw materials containing $\mathrm{Ni}$ is also rising due to the development of various Ni-based alloys. With increasing price and supply risks, an alternative would be to recycle the valuable metal from industrial wastes. Based on the pyrometallurgical extraction process, the Ni-containing alloy scraps and discarded batteries have been utilized as an additional $\mathrm{Ni}$ source in the $\mathrm{Ni}$ slag-cleaning process. ${ }^{9,10}$ The dried Ni-containing concentrates are fed into a $\mathrm{Ni}$ flash smelting furnace, and then the smelting slag with shredded scrap can be reduced by cokes and coal in an electric slag-cleaning furnace. Under the reducing condition, the $\mathrm{Ni}$ is recovered with other elements, such as $\mathrm{Fe}, \mathrm{Co}, \mathrm{Cu}, \mathrm{C}$, and $\mathrm{S} .{ }^{10}$ In order to precisely control the distribution ratio of alloying elements and impurities between the $\mathrm{FeO}_{\mathrm{x}}-\mathrm{SiO}_{2}$ slag and the liquid alloy, the thermodynamic properties of the liquid $\mathrm{Fe}-\mathrm{Ni}$-C alloy have to be understood as one of the core alloy systems. 
Therefore, in the present study, liquid phases of the $\mathrm{Fe}-\mathrm{Ni}$ and $\mathrm{Fe}-\mathrm{Ni}-\mathrm{C}$ systems were thermodynamically assessed. In order to provide the critical input values for the thermodynamic modeling, key experiments were carried out in both the Fe-Ni and $\mathrm{Fe}$ $\mathrm{Ni}-\mathrm{C}$ systems. The melting points of the $\mathrm{Fe}-\mathrm{Ni}$ binary alloy were measured over a wide $\mathrm{Ni}$ concentration range from pure $\mathrm{Ni}$ to Ni-30 wt.\% Fe using differential scanning calorimetry (DSC) under purified $\mathrm{Ar}$ atmosphere. The present experimental results of transition temperatures and the reported enthalpy of mixing data ${ }^{11-14}$ specifically revealed the asymmetry in the Fe-Ni melt. However, although the conventional random mixing model based on the ideal configurational entropy has not been able to properly describe the asymmetry of a liquid solution, it has been considered as an ideal solution ${ }^{15,16}$ because of its very small enthalpy of a mixing value less than $-5 \mathrm{~kJ} / \mathrm{mol}$. On the other hand, in the authors' recent studies, ${ }^{9,17}$ it has been shown that the asymmetry of liquid solution can be precisely described by taking into account the shortrange ordering (SRO) using the modified quasichemical model (MQM) in pair approximation. ${ }^{18}$ Thus, the MQM was adopted for the description of asymmetric liquid solution properties in this study. The reassessed Gibbs free energy of the Fe-Ni liquid solution was merged with the recent MQM results $^{9,19}$ in the binary $\mathrm{Fe}-\mathrm{C}$ and $\mathrm{Ni}-\mathrm{C}$ systems. The $\mathrm{C}$ solubility measured in the present study at $1500^{\circ} \mathrm{C}$ and $1600^{\circ} \mathrm{C}$, and the reported partial enthalpy of mixing of $\mathrm{C}$ in the ternary Fe-Ni-C system, were simultaneously reproduced for the first time with only one constant ternary MQM parameter.

\section{EXPERIMENTAL}

\section{Measurement of Liquidus Temperature in the Fe-Ni System}

The master alloy was produced using a tungsten arc furnace under a purified Ar atmosphere. For this, $30 \mathrm{~g}$ of electrolytic $\mathrm{Fe}(99.99 \%$ purity) and metallic $\mathrm{Ni}$ (99.99\% purity) were mixed according to the desired concentration of the Ni-rich alloys from pure Ni to Ni-30 wt.\% Fe. After melting under the high-purity Ar gas (purity level 6.0), the liquid alloys were homogenized for $120 \mathrm{~min}$ and then quenched rapidly by blowing helium gas. The master alloys were carefully cut and polished for the DSC analysis (NETZSCH STA 449 F3). Around $50 \mathrm{mg}$ of the sample was charged in an alumina crucible [outer diameter (OD): 6.8 , inner diameter (ID): $6 \mathrm{~mm}$, height $(\mathrm{H}): 4 \mathrm{~mm}$ ] capped with an alumina lid. In our preliminary runs of the calorimetric analysis, the use of $\mathrm{Ti}$ or $\mathrm{Zr}$ getters mounted on the sample carrier was not effective to prevent the oxidation of the alloys in the high-temperature region due to the strong interaction between the alloys and O. Thus, purified Ar gas was used in the present study. The Ar gas was first passed through silica gel and phosphorus pentoxide $\left(\mathrm{P}_{2} \mathrm{O}_{5}\right)$ to remove moisture, and ascarite (sodium hydroxidecoated silica) to remove $\mathrm{CO}_{2}$, and then blown into the pre-heating resistance furnace charged with $\mathrm{Mg}$ wires. The $\mathrm{Mg}$ turning furnace was heated to $500^{\circ} \mathrm{C}$ for the best oxidation conditions of $\mathrm{Mg}$ for consuming the $\mathrm{O}_{2}$ in the Ar gas. Such dehydrated and deoxidized $\mathrm{Ar}$ gas was directly blown into the DSC chamber as both a purge gas $(20 \mathrm{~mL} / \mathrm{min})$ and a protective gas $(30 \mathrm{~mL} / \mathrm{min})$. No oxidation was observed during the analysis, which was confirmed by the constant value of the thermogravimetric (TG) curve as shown in Fig. 1 . The sample mass of $\mathrm{Ni}$ 30 wt.\% Fe was changed within $\pm 0.25 \%$ during heating and cooling. The temperature profile (dotted line) was also plotted along with the TG curve (solid line). Two heating and cooling cycles were run to check the reproducibility of the DSC analysis for each sample. Between the heating and cooling segments at $1480^{\circ} \mathrm{C}$ and $1300^{\circ} \mathrm{C}$, the sample was stabilized and homogenized for $15 \mathrm{~min}$. The transition points of the samples were measured by their exothermic or endothermic peaks at the onset point of the phase transformation. The heating and cooling rate was $10^{\circ} \mathrm{C} / \mathrm{min}$.

\section{Measurement of the Carbon Solubility in the Fe-Ni-C Melt}

The $\mathrm{C}$ solubility in the $\mathrm{Fe}-\mathrm{Ni}$ alloy melt was measured over the entire range of melt composition at $1500^{\circ} \mathrm{C}$ and $1600^{\circ} \mathrm{C}$ by continuous alloy addition and sampling techniques. In order to avoid the formation of excess graphite, the experiments were directed at increasing the $\mathrm{C}$ solubility by the addition of high-purity electrolytic iron $(99.99 \%$ purity) into the pure Ni melt for the Ni-rich side or pre-melted Fe-40 wt.\% $\mathrm{Ni}$ alloy for the Fe-rich corner, respectively. Two hundred grams of the alloy charged in a graphite crucible (OD: $60 \mathrm{~mm}$, ID: $54 \mathrm{~mm}, \mathrm{H}: 90 \mathrm{~mm}$ ) was melted using a high-frequency induction furnace $(15 \mathrm{~kW} / 30 \mathrm{kHz})$ under an $\mathrm{Ar}$ atmosphere. The R-type thermocouple $(\mathrm{Pt} / \mathrm{Pt}$ 13 wt.\% Rh) sheathed with closed one end alumina

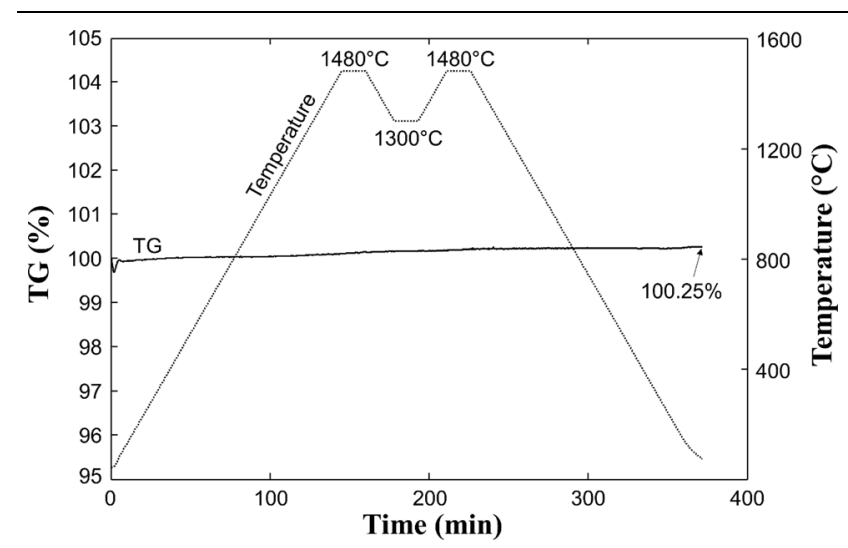

Fig. 1. The programmed temperature profile (dotted line) and corresponding TG analysis of $\mathrm{Ni}-30$ wt.\% Fe sample under the purified Ar gas atmosphere (solid line). 
tube (OD: $6 \mathrm{~mm}$ ) was immersed in the melt for the direct measurement of the melt temperature. After each addition of $\mathrm{Fe}$, the melt was equilibrated within $2 \mathrm{~h}$, which was confirmed by the in situ analysis using a C/S analyzer (Eltra CS-800). The metal samples were extracted using a quartz tube (OD: $6 \mathrm{~mm}$, ID: $4 \mathrm{~mm}$ ) connected to a syringe $(10 \mathrm{~mL})$, and then quenched in ice water within $2 \mathrm{~s}$. The extracted metallic samples were polished to remove the excess graphite and the oxide layer formed on the surface during cooling and solidification. The $\mathrm{Fe}$ and Ni contents were analyzed by using inductively coupled plasma-optical emission spectroscopy (SPECTRO ARCOS).

\section{THERMODYNAMIC MODELING}

The asymmetry of the liquid solution in the binary $\mathrm{Fe}-\mathrm{Ni}$ and ternary $\mathrm{Fe}-\mathrm{Ni}-\mathrm{C}$ systems was thermodynamically analyzed using the MQM in the pair approximation by taking into account the SRO exhibited in the liquid solution. Gibbs energies of pure liquid $\mathrm{Fe}, \mathrm{Ni}$, and $\mathrm{C}$ were taken from the Scientific Group Thermodata Europe (SGTE) data compilation by Dinsdale. ${ }^{20}$ All calculations and assessments in the present study were performed with the FactSage thermochemistry software for using the MQM. ${ }^{21}$

In the MQM, the pair exchange reaction in a binary A-B liquid solution can be expressed as:

$$
(\mathrm{A}-\mathrm{A})+(\mathrm{B}-\mathrm{B})=2(\mathrm{~A}-\mathrm{B}) ; \quad \Delta \mathrm{g}_{\mathrm{AB}}
$$

where $(i-j)$ represents the first-nearest neighbor (FNN) pair and $\Delta \mathrm{g}_{\mathrm{AB}}$ is the Gibbs free energy change for the formation of two moles of (A-B) pairs, respectively. Based on the pair approximation, the following mass balances are considered to account the pair distribution:

$$
\begin{aligned}
& Z_{\mathrm{A}} n_{\mathrm{A}}=2 n_{\mathrm{AA}}+n_{\mathrm{AB}} \\
& Z_{\mathrm{B}} n_{\mathrm{B}}=2 n_{\mathrm{BB}}+n_{\mathrm{AB}}
\end{aligned}
$$

where $n_{i}, n_{i j}$, and $Z_{i}$ are the number of moles of component $i$, the $(i-j)$ pair, and the coordination number of $i$, respectively.

The Gibbs free energy of the liquid solution is given by:

$$
G=\left(n_{\mathrm{A}} g_{\mathrm{A}}^{\circ}+n_{\mathrm{B}} g_{\mathrm{B}}^{\circ}\right)-T \Delta S^{\text {config }}+\left(n_{\mathrm{AB}} / 2\right) \Delta g_{\mathrm{AB}}
$$

where $g_{i}^{\circ}$ is the molar Gibbs free energy of the pure liquid phase which can be directly taken from SGTE. ${ }^{20} \Delta S^{\text {config }}$ is an approximate expression for the configurational entropy of mixing with considering the random distribution of the $(\mathrm{A}-\mathrm{A}),(\mathrm{B}-\mathrm{B})$, and (A-B) pairs on the basis of the ideal entropy of mixing in the one-dimensional Ising approximation: ${ }^{18}$

$$
\begin{aligned}
\Delta S^{\text {config }}= & -\mathrm{R}\left(n_{\mathrm{A}} \ln X_{\mathrm{A}}+n_{\mathrm{B}} \ln X_{\mathrm{B}}\right) \\
& -\mathrm{R}\left[n_{\mathrm{AA}} \ln \left(\frac{X_{\mathrm{AA}}}{Y_{\mathrm{A}}^{2}}\right)+n_{\mathrm{BB}} \ln \left(\frac{X_{\mathrm{BB}}}{Y_{\mathrm{B}}^{2}}\right)\right. \\
& \left.+n_{\mathrm{AB}} \ln \left(\frac{X_{\mathrm{AB}}}{2 Y_{\mathrm{A}} Y_{\mathrm{B}}}\right)\right]
\end{aligned}
$$

where $X_{i}$ and $X_{i j}$ are the mole fraction of $i$ and the pair fraction of the $(i-j)$ pair, respectively. $Y_{i}$ is the coordination equivalent fraction of $i$ calculated from the pair fractions, $Y_{i}=X_{i i}+X_{i j} / 2$.

The $\Delta g_{\mathrm{AB}}$ in Eq. 4 can be expressed in terms of the pair fractions:

$$
\Delta g_{\mathrm{AB}}=\Delta g_{\mathrm{AB}}^{\circ}+\sum_{i \geq 1} g_{\mathrm{AB}}^{i 0} X_{\mathrm{AA}}^{i}+\sum_{j \geq 1} g_{\mathrm{AB}}^{0 j} X_{\mathrm{BB}}^{j}
$$

where $\Delta g_{\mathrm{AB}}^{\circ}, g_{\mathrm{AB}}^{i 0}$ and $g_{\mathrm{AB}}^{0 j}$ are the model parameters to be optimized as a functions of temperature in the A-B binary liquid solution.

The equilibrium pair distribution is calculated under the condition of

$$
\left(\partial G / \partial n_{\mathrm{AB}}\right)_{n_{\mathrm{A}}, n_{\mathrm{B}}}=0
$$

According to a given $\Delta g_{\mathrm{AB}}$ value during the optimization, a different $n_{\mathrm{AB}}$ can be obtained as a solution of Eq. 7, then $n_{\mathrm{AA}}$ and $n_{\mathrm{BB}}$ can be evaluated by Eqs. 2 and 3 with the coordination number information. In order to reproduce all available liquid solution properties, the optimum $\Delta g_{\mathrm{AB}}$ values are repeatably searched.

In this model, the asymmetry of the liquid solution can be represented as a non-equimolar composition of maximum SRO in each binary system by setting the ratio of the coordination numbers, $Z_{\mathrm{B}} / Z_{\mathrm{A}}$ from the following equations:

$$
\begin{aligned}
& \frac{1}{Z_{\mathrm{A}}}=\frac{1}{Z_{\mathrm{AA}}^{\mathrm{A}}}\left(\frac{2 n_{\mathrm{AA}}}{2 n_{\mathrm{AA}}+n_{\mathrm{AB}}}\right)+\frac{1}{Z_{\mathrm{AB}}^{\mathrm{A}}}\left(\frac{n_{\mathrm{AB}}}{2 n_{\mathrm{AA}}+n_{\mathrm{AB}}}\right) \\
& \frac{1}{Z_{\mathrm{B}}}=\frac{1}{Z_{\mathrm{BB}}^{\mathrm{B}}}\left(\frac{2 n_{\mathrm{BB}}}{2 n_{\mathrm{BB}}+n_{\mathrm{AB}}}\right)+\frac{1}{Z_{\mathrm{BA}}^{\mathrm{B}}}\left(\frac{n_{\mathrm{AB}}}{2 n_{\mathrm{BB}}+n_{\mathrm{AB}}}\right)
\end{aligned}
$$

where $Z_{\mathrm{AA}}^{\mathrm{A}}$ and $Z_{\mathrm{AB}}^{\mathrm{A}}$ are the values of $Z_{A}$, when all the nearest neighbors of an $\mathrm{A}$ are $\mathrm{A}$ and $\mathrm{Bs}$, respectively, and $Z_{\mathrm{BB}}^{\mathrm{B}}$ and $Z_{\mathrm{BA}}^{\mathrm{B}}$ are defined vice versa. In the present study, the coordination numbers of pure elements, $Z_{\mathrm{FeFe}}^{\mathrm{Fe}}, Z_{\mathrm{NiNi}}^{\mathrm{Ni}}$, and $Z_{\mathrm{CC}}^{\mathrm{C}}$ were set to 6 , while the coordination number ratio, $Z_{\mathrm{FeNi}}^{\mathrm{Fe}} / Z_{\mathrm{FeNi}}^{\mathrm{Ni}}$ was set to $2(=6 / 3)$ to describe the asymmetry of the liquid solution with the maximum SRO near $X_{\mathrm{Ni}}=0.6-0.7$ in the $\mathrm{Fe}-\mathrm{Ni}$ system. ${ }^{5,6,11-14}$ The values of the coordination numbers selected in the Fe-Ni-C system are listed in Table I.

After optimizing the binary $\mathrm{Fe}-\mathrm{Ni}$ system, the Gibbs free energy of the ternary Fe-Ni-C liquid phase is estimated by merging it with the new MQM parameters in the Fe-C ${ }^{19}$ and Ni-C systems. ${ }^{9}$ In the ternary system, the Toop-like interpolation method 
Table I. Optimized model parameters in the Fe-Ni-C system

Liquid (L)

MQM: (Fe,Ni,C)

$Z_{\mathrm{FeFe}}^{\mathrm{Fe}}=Z_{\mathrm{NiNi}}^{\mathrm{Ni}}=Z_{\mathrm{CC}}^{\mathrm{C}}=6$ [This work]

$Z_{\mathrm{FeNi}}^{\mathrm{Fe}}=6, Z_{\mathrm{FeNi}}^{\mathrm{Ni}}=3$ [This work]

$Z_{\mathrm{FeC}}^{\mathrm{C}}=6, Z_{\mathrm{FeC}}^{\mathrm{Fe}}=3[19]$

$Z_{\mathrm{NiC}}^{\mathrm{C}}=8, Z_{\mathrm{NiC}}^{\mathrm{Ni}}=2[9]$

$g_{\mathrm{Fe}(1)}^{\circ}, g_{\mathrm{Ni}(1)}^{\circ}, g_{\mathrm{C}(1)}^{\circ}$

$\Delta g_{\mathrm{FeNi}}$

$\Delta g_{\mathrm{FeC}}$

$\Delta g_{\mathrm{NiC}}$

$g_{\mathrm{FeNi}(\mathrm{C})}^{001}$

\author{
$-8368+2.887 T+(1674-1.004 T) X_{\mathrm{FeFe}}$ [This work] \\ $-30,460+3.138 T+(-1130) X_{\mathrm{FeFe}}[19]$ \\ $-24,476+6.339 T+(4812-4.293 T) X_{\mathrm{NiNi}}+(1.979 T) X_{\mathrm{NiNi}}^{2}[9]$ \\ 10,460 [This work]
}

was applied to consider the non-metallic element, with $\mathrm{C}$ as the asymmetric component. The Gibbs free energy of all the solid phases have been modeled using compound energy formalism (CEF) in the Fe-Ni system by Xing et al., ${ }^{15}$ the Fe-C system by Gustafson, ${ }^{22}$ the Ni-C system by Lee ${ }^{23}$ and the Fe-Ni-C system by Gabriel et al. ${ }^{24}$ with high accuracy. Therefore, these stable solid phases were retained to be compatible with previous modeling results because they have been widely used for the prediction of solid phase equilibria in the various multicomponent systems.

\section{RESULTS AND DISCUSSION}

\section{Fe-Ni Binary System}

Figure 2a shows the Fe-Ni phase diagram calculated using the previous modeling results. ${ }^{15,16}$ There are five solution phases in this binary system. The $\delta$ - and $\alpha$-body centered cubic (BCC) phases are stable on the Fe-rich corner, according to the temperature. The wide FCC stable region exists below the melting point with complete solid solubility. At the lower temperature, the ordered FCC phase can be formed on the basis of the $\mathrm{Ni}_{3} \mathrm{Fe}$ structure. Lee ${ }^{16}$ adopted Xing et al.'s ${ }^{15}$ result for the Gibbs energy description of these solid phases, while he reassessed the liquid solution to increase the reproducibility of the transition temperature at the liquid/solid phase boundary in the Fe-rich region. Figure $2 \mathrm{~b}$ shows the enlarged part of the $\mathrm{Fe}-\mathrm{Ni}$ phase diagram near the melting point, together with the selected experimental data mainly for the high $\mathrm{Ni}$ concentration range. ${ }^{5,6,25,26}$ The calculated liquidus/solidus curves by $\mathrm{Lee}^{16}$ were slightly overestimated compared with the experimental results up to $20^{\circ} \mathrm{C}$ at the $\mathrm{Ni}$-rich side by considering the supercooling effect of the measurements. On the other hand, the recent optimization by Ohnuma et al. ${ }^{4}$ determined the Gibbs energy of the liquid solution for the best-fitting curves with the experimental results ${ }^{5,6}$ as shown in Fig. $2 b$.
Hanson and Freeman ${ }^{5}$ and Kase ${ }^{6}$ have measured the liquidus points over the entire composition range in the binary $\mathrm{Fe}-\mathrm{Ni}$ system. Hanson and Freeman ${ }^{5}$ melted the alloys using the commercial Fe (wt.\% $\mathrm{C}=0.012$, wt. $\% \quad \mathrm{Si}=0.017$, wt. $\%$ $\mathrm{S}=0.017$, wt. $\% P=0.014$, wt. $\% \mathrm{Mn}=0.07)$ and $\mathrm{Ni}$ shot (99.98\% purity) under the purified $\mathrm{N}_{2}$ gas. In their study, The arrest points measured by the immersed thermocouple during cooling were considered as the liquidus temperatures. Only cooling experiments were carried out to protect the thermocouple and its sheath by decreasing the exposure time at high temperatures. Kase ${ }^{6}$ also measured the liquidus temperatures over the entire Ni concentration by measuring the arrest point during cooling. Even though he used more contaminated raw materials such as wrought iron (wt.\% $\mathrm{C}=0.054$, wt. $\% \mathrm{Si}=0.188$, wt. $\% \quad \mathrm{~S}=0.008$, wt. $\%$ $P=0.065$, wt. $\% \mathrm{Mn}=0.015$ ) and $\mathrm{Ni}$ shots (wt.\% $\mathrm{C}=0.043$, wt. $\% \mathrm{Mn}=0.179$, wt. $\% \mathrm{Fe}=0.153$ ), his result has a good correlation with Hanson and Freeman's ${ }^{5}$ data. The solidus evaluated by the intersection of two tangents from the cooling curve was not plotted in Fig. 2b. Bennek and Schafmeis$\operatorname{ter}^{25}$ measured the liquidus and solidus temperatures of $\mathrm{Fe}-\mathrm{Ni}$ alloys up to $49.2 \mathrm{wt} . \% \mathrm{Ni}$ by cooling experiments. In order to prevent the supercooling problem reported by the previous studies during cooling, ${ }^{5,6}$ they controlled the cooling temperature of $0.17-2^{\circ} \mathrm{C} / \mathrm{s}$. Also, the cooling data were verified by the separate measurements during heating. However, there was no significant difference compared with Hanson and Freeman ${ }^{5}$ and Kase's ${ }^{6}$ results. Later, Kundrat ${ }^{26}$ measured the liquidus temperature in the Fe-rich side up to 24.9 wt.\% Ni using a differential thermal analysis (DTA). With the calibration of the thermocouples against the melting point of $\mathrm{Fe}\left(1538^{\circ} \mathrm{C}\right), \mathrm{Ni}\left(1455^{\circ} \mathrm{C}\right)$, and $\mathrm{Ag}\left(961^{\circ} \mathrm{C}\right)$, he confirmed the analytical error of $\pm 1^{\circ} \mathrm{C}$ caused by the thermal gradient during cooling. Since his DTA data at the Fe-rich side were much higher than the previous results, ${ }^{5,6,25}$ it was worthwhile to check the 

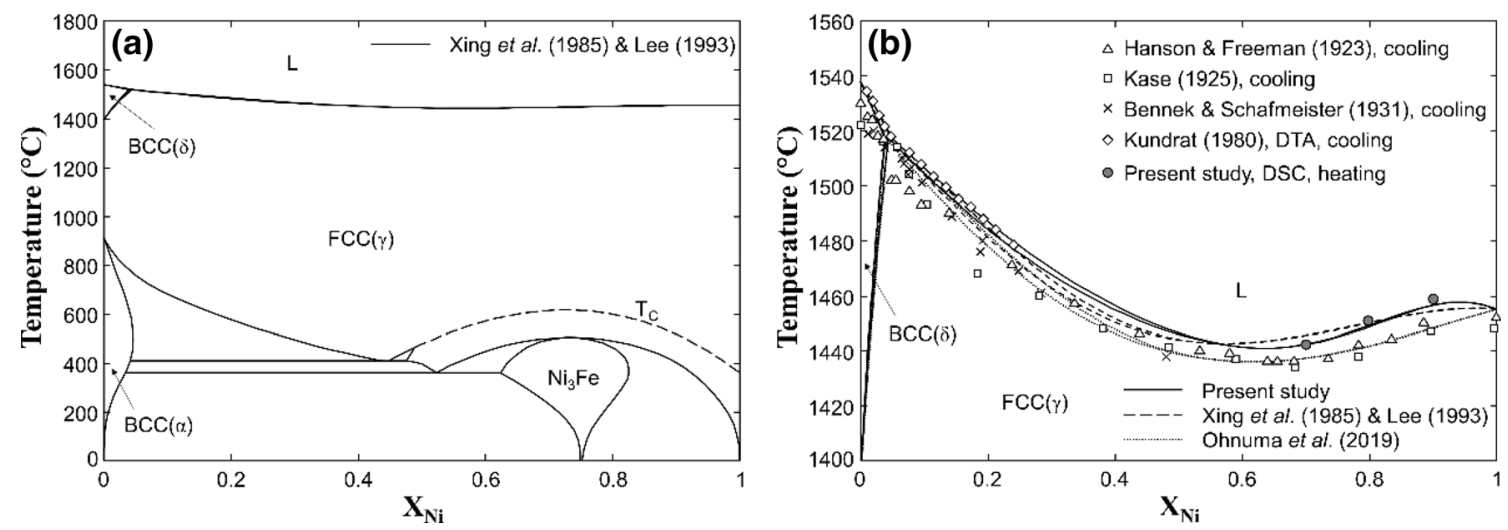

Fig. 2. (a) Calculated phase diagram of the Fe-Ni system, ${ }^{15,16}$ and (b) the enlarged part near the melting temperature along with the previous experimental data; Tc Curie temperature.

accuracy of the earlier data measured in the $1920 \mathrm{~s}^{5,6}$ at the Ni-rich side. The highly sensitive DSC was used without any contamination of alloys, undercooling effect, or oxidation problem under the purified Ar atmosphere. The transition temperatures from pure Ni to Ni-30 wt.\% Fe alloy obtained during heating and cooling segments were plotted along with the previous studies, as shown in Fig. 2b. The present results during heating were much closer to the predicted liquidus line by Lee's ${ }^{16}$ optimization. The data during cooling were not used in the present study due to the significant undercooling up to $12^{\circ} \mathrm{C}$.

In order to simultaneously reproduce the present experimental results of high-Ni alloys and Kundrat's $^{26}$ data at the Fe-rich side, the $\Delta g_{\mathrm{FeNi}}^{\circ}$ value was first determined. Then, the first-order parameter of the $(\mathrm{Fe}-\mathrm{Fe})$ pair, $g_{\mathrm{FeNi}}^{10}$ was adjusted with the temperature-dependent term to keep the reported peritectic temperature and composition at the $\mathrm{Fe}$ rich side. ${ }^{26}$ By setting the coordination number ratio of $Z_{\mathrm{FeNi}}^{\mathrm{Fe}} / Z_{\mathrm{FeNi}}^{\mathrm{Ni}}=2(=6 / 3)$, the composition-dependent parameter of the $(\mathrm{Ni}-\mathrm{Ni})$ pair, $g_{\mathrm{FeNi}}^{01}$ was neglected. Since the non-configurational entropic effect by the SRO of the $\mathrm{Fe}$ and $\mathrm{Ni}$ atoms was intrinsically considered by Eq. 5, the change of the coordination number ratio was very effective to describe the minimum melting temperature near $X_{\mathrm{Ni}}=0.7$ at the Ni-rich side without excess enthalpy and entropy of mixing for the (Ni-Ni) pair. The stability of this asymmetric liquid solution is evident in the Ni-rich region. It should also be noted that the minimum liquidus temperature corresponded well with the basic composition of the ordered FCC phase $\left(X_{\mathrm{Ni}}=0.75\right)$.

Figure $3 \mathrm{a}$ and $\mathrm{b}$ shows the calculated and measured integral and partial enthalpies of mixing in the $\mathrm{Fe}-\mathrm{Ni}$ alloy melt, respectively. As shown in Fig. 3a, the measured enthalpy of mixing is significantly scattered, ${ }^{11-14}$ while all the data show the definite asymmetry. The integral enthalpy of mixing reached the minimum value in the range of $X_{\mathrm{Ni}}$ from 0.6 to 0.7 , but the absolute value of enthalpy of mixing is very small, less than $-5 \mathrm{~kJ} / \mathrm{mol}$ at the minimum point. Therefore, the contribution of enthalpy to the Gibbs free energy of mixing can be omitted in the high-temperature region according to the Gibbs energy function, $\Delta G=\Delta H-T \Delta S$. That is the reason why the $\mathrm{Fe}-\mathrm{Ni}$ liquid solution has been considered as an ideal solution. Since the composition-dependent parameters exert a stronger effect on the overall liquid solution properties in such narrow energy levels and heat content range, it is very important to reduce the number of parameters and to minimize the value itself. By setting the asymmetric SRO composition, the present model can explain the asymmetric enthalpy of mixing reasonably without composition-dependent parameters for the Ni-rich side. As shown in Fig. 3b, the partial enthalpy of mixing evaluated from the integral enthalpy by Batalin et al. ${ }^{13}$ and Iguchi et al. ${ }^{14}$ was reproduced very well. The intersecting point of partial enthalpy of $\mathrm{Fe}$ and $\mathrm{Ni}$ also represents the asymmetry of the liquid solution.

\section{Fe-Ni-C Ternary System}

The validity of reassessed binary Fe-Ni liquid solution was confirmed by extending the system to the Fe-Ni-C system, because the asymmetry of the Fe-Ni liquid solution was remarkably shown for describing the partial properties of $\mathrm{C}$ in the ternary alloy melt, such as the $\mathrm{C}$ solubility and the partial enthalpy of mixing of C. Also, it was appropriate to explain the temperature effect on the asymmetry due to the lower melting points by the addition of $\mathrm{C}$ in the Fe-Ni melt. Moreover, no stable carbide is formed in spite of the strong affinity of $\mathrm{C}$ with $\mathrm{Fe}$ and $\mathrm{Ni}$ in the $\mathrm{Fe}-\mathrm{Ni}-\mathrm{C}$ system.

This ternary system has been optimized by Gabriel et al. ${ }^{24}$ using the random mixing model for the liquid solution and CEF for the solid phases, respectively. They extensively reviewed the available thermodynamic data. According to their evaluations, there are four solution phases, liquid, FCC, $\mathrm{BCC}$, and $(\mathrm{Fe}, \mathrm{Ni})_{3} \mathrm{C}$ cementite. As mentioned earlier, the present study only adopted the Gibbs free 

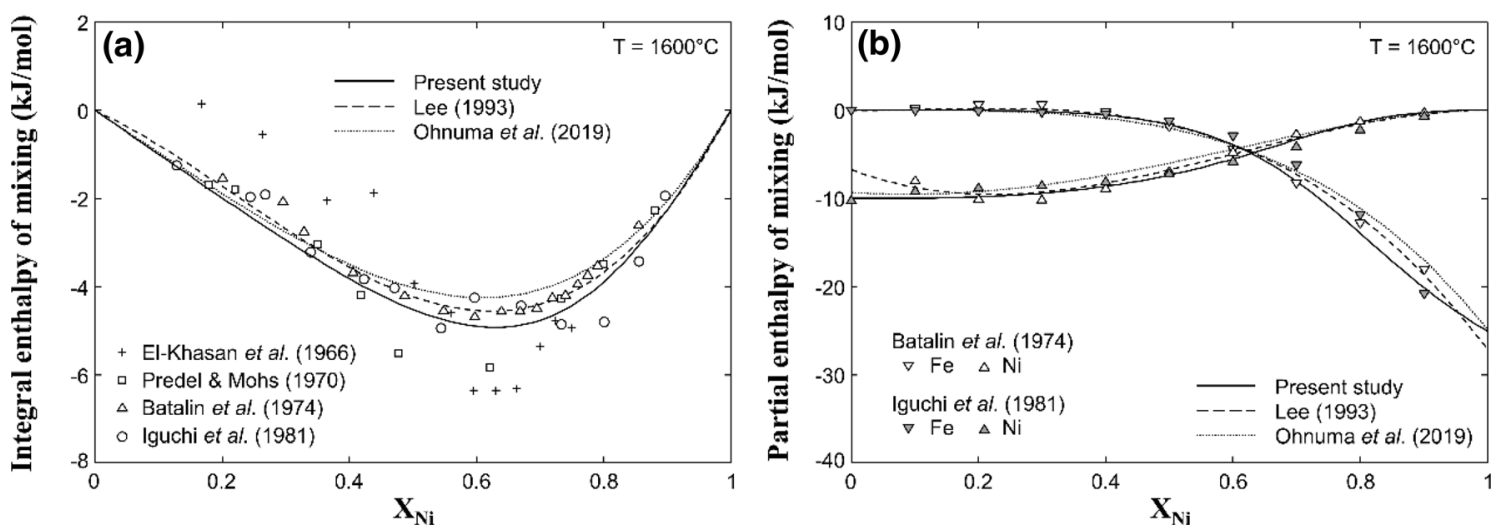

Fig. 3. (a) Integral and (b) partial enthalpy of mixing in liquid Fe-Ni alloy at $1600^{\circ} \mathrm{C}$.

energy description of the solid solution phases from the previous assessments ${ }^{15,22-24}$ and reassessed the liquid solution by the MQM. For the optimization of the liquid solution in this ternary system, the binary MQM parameters of the Fe-C system determined by Shubhank and Kang, ${ }^{19}$ and that of the NiC system by the authors' recent study, ${ }^{9}$ were directly used along with the present $\mathrm{Fe}-\mathrm{Ni}$ binary parameters. The Toop-like interpolation method with $\mathrm{C}$ as the asymmetric component was used because the $\mathrm{Fe}-\mathrm{C}$ and $\mathrm{Ni}-\mathrm{C}$ liquid solutions exhibit considerable negative deviation by the strong attraction force between the metal ( $\mathrm{Fe}$ and $\mathrm{Ni}$ ) and the gaseous impurity (C). In contrast, the liquid solution in the $\mathrm{Fe}-\mathrm{Ni}$ system has only a small deviation from the ideal mixing.

Figure 4 shows the $\mathrm{C}$ solubility in the $\mathrm{Fe}-\mathrm{Ni}-\mathrm{C}$ alloy melts measured by the present study and various researchers ${ }^{27-30}$ in the temperature range from $1300^{\circ} \mathrm{C}$ to $1600^{\circ} \mathrm{C}$. The reported $\mathrm{C}$ solubility data show larger scatters at a higher temperature. This can be caused by the formation of excess graphite during quenching from high temperatures. The excess graphite can also be formed by the addition of $\mathrm{Ni}$ into the $\mathrm{C}$-saturated $\mathrm{Fe}-\mathrm{Ni}$ melt due to the decreasing solubility limit with the $\mathrm{Ni}$ content. Therefore, the $\mathrm{C}$ solubility in $\mathrm{Fe}-\mathrm{Ni}$ alloy melt was reversely measured over the entire concentration range at $1500^{\circ} \mathrm{C}$ and $1600^{\circ} \mathrm{C}$ by adding $\mathrm{Fe}$ into the high-Ni alloys with the rapid quenching technique. The $\mathrm{C}$ solubility in liquid solution decreases with the increasing $\mathrm{Ni}$ content. This indicates that the affinity of $\mathrm{C}$ to $\mathrm{Fe}$ is stronger than that to Ni. However, it has minimum solubility at a particular concentration of the Ni-rich side. In order to reproduce the $\mathrm{C}$ solubility data with the solubility minimum, only one adjustable ternary MQM parameter, $g_{\mathrm{FeNi}(\mathrm{C})}^{001}=10,460$, were introduced to decrease the stability of the liquid solution at around $80 \mathrm{wt} . \% \mathrm{Ni}$. On the other hand, the previous optimization ${ }^{24}$ based on the random mixing model added the considerable values of ternary parameters even with temperature and composition dependence, $\quad L_{\mathrm{C}, \mathrm{Fe}, \mathrm{Ni}}^{\mathrm{liq}}=122,200-58.5 T-20,000($ $\left.X_{\mathrm{Fe}}-X_{\mathrm{Ni}}\right)$.

The minimum solubility of $\mathrm{C}$ in the $\mathrm{Fe}-\mathrm{Ni}-\mathrm{C}$ system has also been observed, by Petrova and Shvartsmann, ${ }^{31}$ in the low-temperature region in the ternary FCC solid solution. They explained that the ordering of $\mathrm{Fe}$ and $\mathrm{Ni}$ near $X_{\mathrm{Ni}}=0.75$ can decrease the number of spaces for occupying $\mathrm{C}$ atoms in the solid lattice. Similarly, the minimum solubility in the liquid solution can be described by the SRO using MQM. The calculated pair fractions of various pairs in the liquid Fe-Ni-C alloy saturated with $\mathrm{C}$ at $1350^{\circ} \mathrm{C}$ are shown in Fig. 5a and b. The equilibrium $\mathrm{C}$ content in the melt can be determined by a sum of $\mathrm{C}$ in various $\mathrm{FNN}$ pairs containing $\mathrm{C}$, such as (Fe-C), (Ni-C), and (C-C) in the MQM calculation. When the $\mathrm{Ni}$ content is low, most $\mathrm{C}$ is represented by the (Fe-C) pairs, and it gradually decreases with increasing Ni content. On the other hand, the (Ni-C) pairs increase with increasing $\mathrm{Ni}$ content. Although the amount of the (C-C) pair is relatively smaller than the (Fe-C) and (Ni-C) pairs, as shown in Fig. 5b, it directly reflects the minimum solubility of $\mathrm{C}$ in the ternary liquid solution. The minimum point of the (C-C) pair fraction corresponded very well with the composition of the minimum $\mathrm{C}$ solubility as well as the maximum (Fe-Ni) fraction, as shown in Fig. 5a. This indicates that the SRO between $\mathrm{Fe}$ and $\mathrm{Ni}$ decreases the stability of the $\mathrm{C}$ dissolved in the liquid solution, and the destabilized $\mathrm{C}$ can be activated to form graphite. Therefore, the liquidus line of graphite can penetrate along the maximum SRO composition of the ( $\mathrm{Fe}-\mathrm{Ni})$ pair in the ternary liquid solution.

Figure 6 shows the variation of the partial enthalpy of mixing of $\mathrm{C}$ in $\mathrm{Fe}-\mathrm{Ni}-\mathrm{C}$ melt. Witusiewicz et al. ${ }^{32-34}$ have measured the partial enthalpy of $\mathrm{C}$ with respect to the graphite reference state using high-temperature isothermal calorimetry. Their data were converted into hypothetical liquid $\mathrm{C}$ based on the enthalpy of fusion of $\mathrm{C}$ from the 

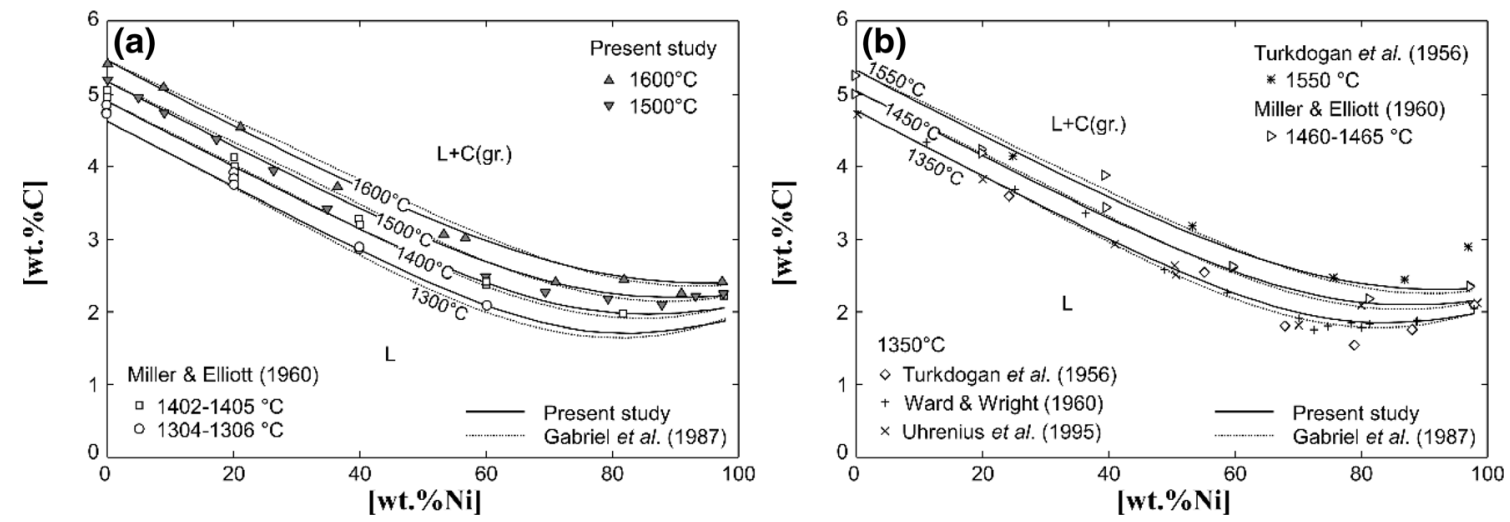

Fig. 4. Calculated $\mathrm{C}$ Solubility in Fe-Ni-C melt along with the present and previous experimental data at $(\mathrm{a}) 1300^{\circ} \mathrm{C}, 1400^{\circ} \mathrm{C}, 1500^{\circ} \mathrm{C}$, and $1600^{\circ} \mathrm{C}$, and (b) $1350^{\circ} \mathrm{C}, 1450^{\circ} \mathrm{C}$, and $1550^{\circ} \mathrm{C}$.
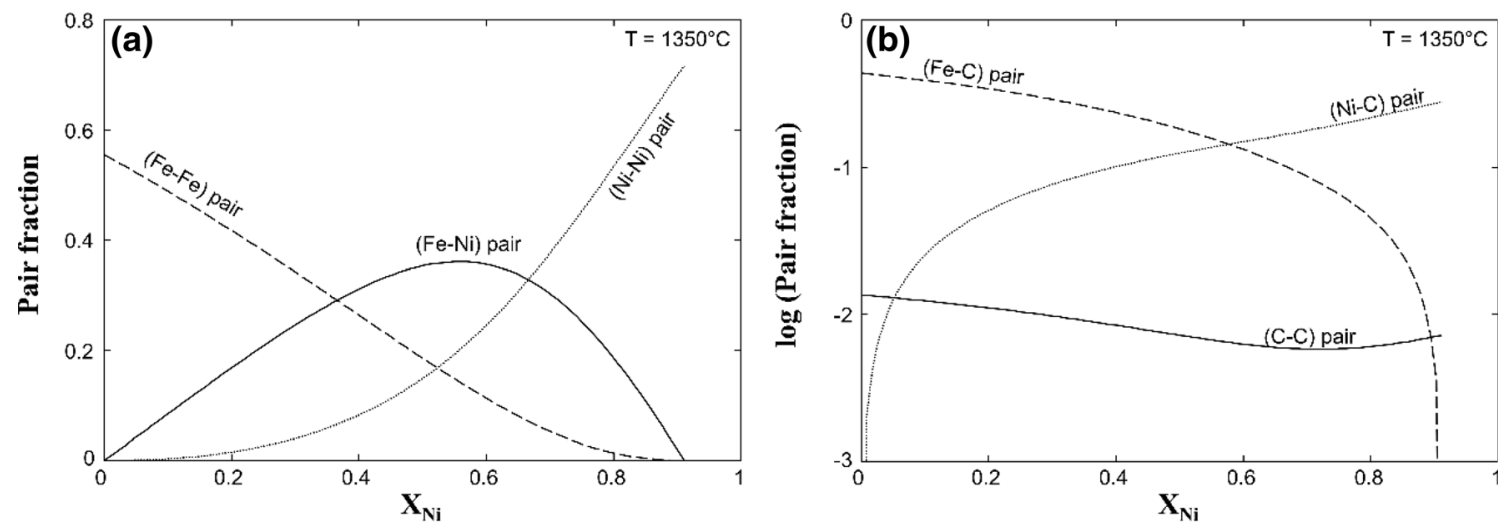

Fig. 5. Calculated pair fractions of (a) (Fe-Fe), (Ni-Ni), and (Fe-Ni), and (b) (Fe-C), (Ni-C), and (C-C) pairs in the C-saturated Fe-Ni alloy melt at $1350^{\circ} \mathrm{C}$

SGTE database. ${ }^{20}$ The accuracy of the partial enthalpy data at the dilute concentration of $\mathrm{C}$ in the binary Fe-C ${ }^{34}$ and $\mathrm{Ni}-\mathrm{C}$ systems ${ }^{33}$ was discussed and confirmed by the authors' recent study. ${ }^{9}$ In the ternary $\mathrm{Fe}-\mathrm{Ni}-\mathrm{C}$ system, the maximum point of the partial enthalpy of $\mathrm{C}$ was observed in the Ni-rich corner, and the present model calculation successfully reproduced the asymmetric tendency of the experimental data within the error range. It should be noted that the maximum composition of partial enthalpy corresponded well with the $\mathrm{Ni}$ concentration of the minimum $\mathrm{C}$ solubility (Fig. 4). However, the previous optimization by the random mixing model $^{24}$ was much underestimated compared with the experimental data, particularly at Ni-rich side. Since there was no significant difference in the predicted $\mathrm{C}$ solubility between the present and previous calculations, ${ }^{24}$ as shown in Fig. 4, this proves that the individual contribution of the enthalpy and entropy to the Gibbs energy of the liquid solution is better represented by the present study using MQM.

Figure 7 shows the predicted liquidus projection and the iso-enthalpy of mixing in the Fe-Ni-C system using the parameters determined in the present study. In the liquidus projection (Fig. 7a),

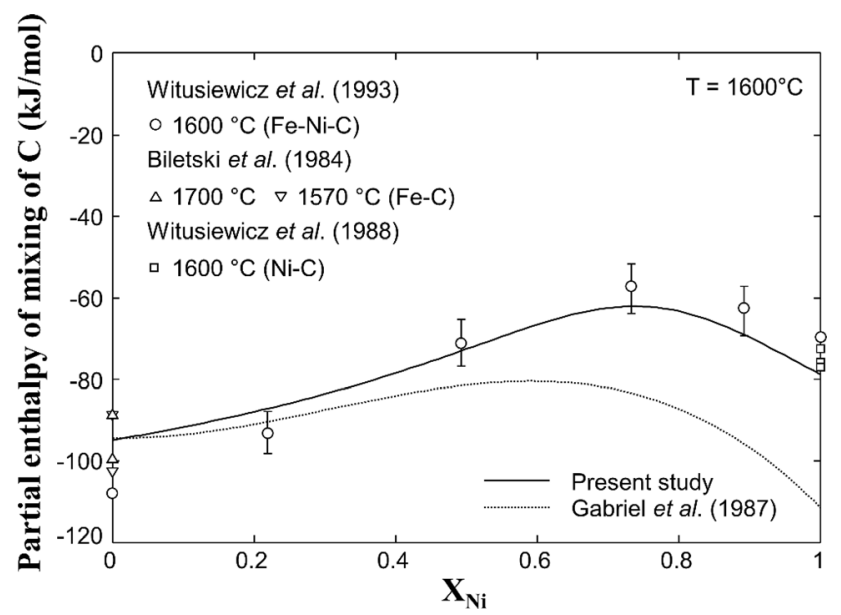

Fig. 6. Calculated partial enthalpy of mixing of $\mathrm{C}$ in Fe-Ni-C melt at infinite dilution of $\mathrm{C}$ at $1600^{\circ} \mathrm{C}$.

the bold line indicates the univariant and the colored lines are the isotherms calculated every $100^{\circ} \mathrm{C}$. This diagram can be used to track the proper temperature for melting the $\mathrm{Fe}-\mathrm{Ni}-\mathrm{C}$ alloy depending on the concentration. As shown in Fig. 7b, the iso-enthalpy curves with respect to pure liquid $\mathrm{Fe}$, $\mathrm{Ni}$, and hypothetical liquid $\mathrm{C}$ show the significant 

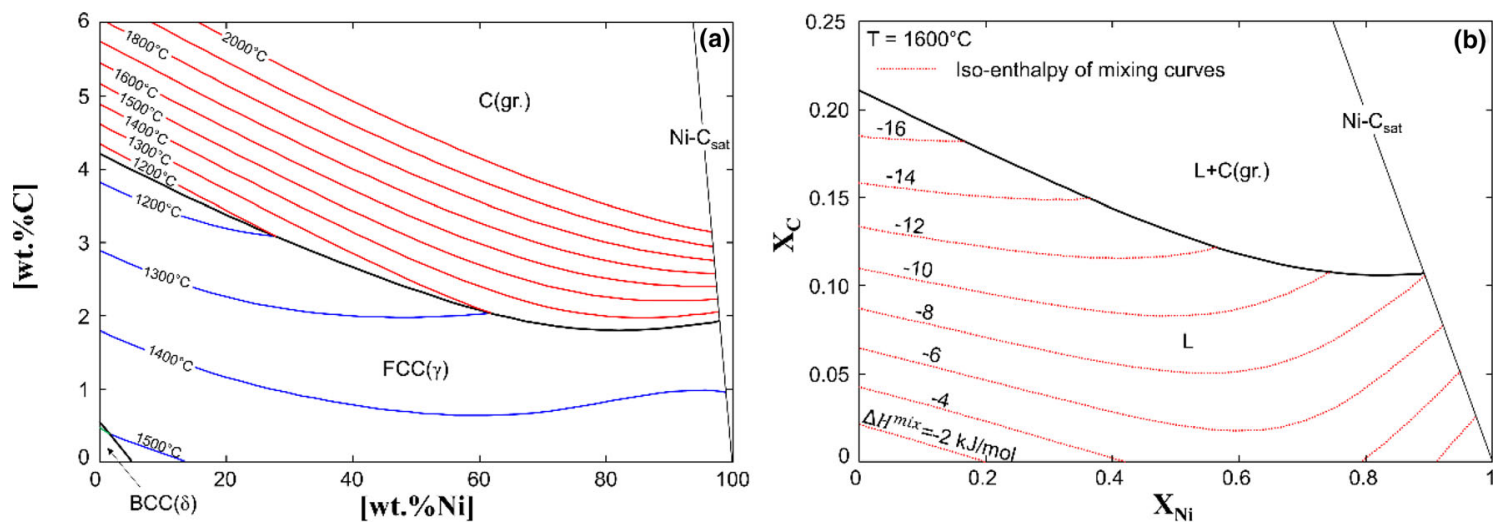

Fig. 7. Projections of (a) liquidus and (b) enthalpy of mixing in the ternary Fe-Ni-C system.

inflection in the Ni-rich region. Such information is also useful to predict the heat loss of the liquid $\mathrm{Ni}$ alloy in the furnace and heat transfer between the slag and alloy during the pyrometallurgical extraction process, as well as the refining process of high$\mathrm{Ni}$ alloy production.

\section{CONCLUSION}

The asymmetry of the Fe-Ni and Fe-Ni-C liquid solutions was thermodynamically described using the MQM. The liquidus points of Ni-rich side in the $\mathrm{Fe}-\mathrm{Ni}$ system were revised by measuring the transition temperature between the liquid and FCC phases. In the Fe-Ni-C ternary system, the present optimization reproduced the $\mathrm{C}$ solubility and partial enthalpy of mixing of $\mathrm{C}$, simultaneously. The solubility minimum of $\mathrm{C}$ and the maximum partial enthalpy of $\mathrm{C}$ were strongly related to the SRO of the binary Fe-Ni liquid solution. This shows the importance of accurate description in the basic binary system for the extendibility and reproducibility in multicomponent systems. The present model parameters can provide more precise information, such as heat content change and its temperature dependence, not only for the pyrometallurgical extraction process of $\mathrm{Ni}$ but also for the high-Ni alloy production.

\section{ACKNOWLEDGEMENTS}

Funding from the Business Finland, SYMMET project (Grant Number 3891/31/2018) is greatly appreciated. This study utilized the Academy of Finland's RawMatTERS Finland Infrastructure (RAMI), based jointly at Aalto University, GTK and VTT.

\section{CONFLICT OF INTEREST}

The authors declare that they have no conflict of interest.

\section{OPEN ACCESS}

This article is licensed under a Creative Commons Attribution 4.0 International License, which permits use, sharing, adaptation, distribution and reproduction in any medium or format, as long as you give appropriate credit to the original author(s) and the source, provide a link to the Creative Commons licence, and indicate if changes were made. The images or other third party material in this article are included in the article's Creative Commons licence, unless indicated otherwise in a credit line to the material. If material is not included in the article's Creative Commons licence and your intended use is not permitted by statutory regulation or exceeds the permitted use, you will need to obtain permission directly from the copyright holder. To view a copy of this licence, visit http://creativecom mons.org/licenses/by/4.0/.

\section{REFERENCES}

1. J.H. Weber and M.K. Banerjee, Encyclopedia of Materials: Science and Technology (Amsterdam: Elsevier, 2001).

2. Z.K. Liu, Y. Wang, and S. Shang, Sci. Rep. 4, 7043 (2014).

3. J. Liu, L.J. Riddiford, C. Floristean, F. Goncalves-Netoa, M. Rezaeeyazdi, L.H. Lewis, and K. Barmak, J. Alloys Compd. 689, 593 (2016).

4. I. Ohnuma, S. Shimenouchi, T. Omori, K. Ishida, and R. Kainuma, CALPHAD 67, 101677 (2019).

5. D. Hanson and J.R. Freeman, J. Iron Steel Inst. 167, 301 (1923).

6. T. Kase, Sci. Rep. Tohoku Imp. Univ. 14, 173 (1925).

7. L.B. Chen, R. Wei, K. Tang, J. Zhang, F. Jiang, L. He, and J. Sun, Mater. Sci. Eng. A 716, 150 (2018).

8. Y. Xu, Q. Jin, X. Xiao, X. Cao, G. Jia, Y. Zhu, and H. Yin, Mater. Sci. Eng. A 528, 4600 (2011).

9. J.M. Jeon, S.Y. Kwon, D. Lindberg, M.K. Paek, Metall. Mater. Trans. B (2021). https://doi.org/10.1007/s11663-02001995-6.

10. R. Ruismäki, A. Dańczak, L. Klemettinen, P. Taskinen, D. Lindberg, and A. Jokilaakso, Minerals 10, 435 (2020).

11. A. El-Khasan, K. Abdel-Aziz, A.A. Vertman, and A.M. Samarin, Izv. Akad. Nauk. SSSR Met. 3, 19 (1966).

12. V.B. Predel and R. Mohs, Arch. Eisenhüttenwes. 41, 143 (1970).

13. G.I. Batalin, N.N. Minenko, and V.S. Sundavtsova, Russ. Metall. 5, 82 (1974). 
14. Y. Iguchi, Y. Tozaki, M. Kakizaki, T. Fuwa, and S. Ban-ya, Tetsu-to-Hagané 67, 925 (1981).

15. Z.S. Xing, D.D. Gohil, A.T. Dinsdale, T. Chart, $D M A(A)$ (National Physical Laboratory, London, 1985), p. 103.

16. B.J. Lee, CALPHAD 17, 251 (1993).

17. M.K. Paek, J.J. Pak, and Y.B. Kang, CALPHAD 46, 92 (2014).

18. A.D. Pelton, S.A. Degterov, G. Eriksson, C. Robelin, and Y. Dessureault, Metall. Mater. Trans. B 31B, 651 (2000).

19. K. Shubhank and Y.B. Kang, CALPHAD 45, 127 (2014).

20. A.T. Dinsdale, CALPHAD 15, 317 (1991).

21. C.W. Bale, E. Bélisle, P. Chartrand, S.A. Decterov, G. Eriksson, A.E. Gheribi, K. Hack, I.H. Jung, Y.B. Kang, J. Melançon, A.D. Pelton, S. Petersen, C. Robelin, J. Sangster, P. Spencer, and M.A. Van Ende, CALPHAD 54, 35 (2016).

22. P. Gustafson, Scand. J. Metall. 14, 259 (1985).

23. B.J. Lee, CALPHAD 16, 121 (1992).

24. A. Gabriel, P. Gustafson, and I. Ansara, CALPHAD 11, 203 (1987).

25. H. Bennek and P. Schafmeister, Arch. Eisenhüttenwes. 5, 123 (1931).

26. D.M. Kundrat, Thesis, Massachusetts Institute of Technology, January 1980.
27. E.T. Turkdogan, R.A. Hancock, S.I. Herlitz, and J. Dentan, J. Iron Steel Inst. 183, 69 (1956).

28. K.O. Miller and J.F. Elliott, Trans. Metall. Soc. AIME 218, 900 (1960)

29. R.G. Ward and J.A. Wright, J. Iron Steel Inst. 194, 304 (1960).

30. B. Uhrenius, K. Forsén, B.O. Haglund, and I. Andersson, J. Phase Equilib. 16, 430 (1995).

31. E.F. Petrova and L.A. Shvartsmann, Dokl. Akad. Nauk SSSR 146, 646 (1962).

32. V.T. Witusiewich and V.S. Shumikhin, Rasplavy 5, 78 (1993).

33. V.T. Witusiewicz, A.K. Biletski, and V.S. Shumikhin, Metally 4, 61 (1988).

34. A.K. Biletski, V.T. Witusiewicz, and V.S. Shumikin, Metally 6, 40 (1984).

Publisher's Note Springer Nature remains neutral with regard to jurisdictional claims in published maps and institutional affiliations. 